Service social

\title{
Lecture de la recherche sur les problèmes sociaux
}

\section{Richard Lefrançois}

Volume 39, numéro 2, 1990

Les problèmes sociaux

URI : https://id.erudit.org/iderudit/706481ar

DOI : https://doi.org/10.7202/706481ar

Aller au sommaire du numéro

Éditeur(s)

École de service social de l'Université Laval

ISSN

1708-1734 (numérique)

Découvrir la revue

Citer cet article

Lefrançois, R. (1990). Lecture de la recherche sur les problèmes sociaux. Service social, 39(2), 129-140. https://doi.org/10.7202/706481ar

\section{Résumé de l'article}

Procédant à une observation de certaines des tendances qui influencent présentement l'évolution de la recherche sociale au Québec, l'auteur constate que la conjoncture du début des années 1980 a eu son effet sur la perception et la définition des problèmes sociaux. Certains indices comme la résurgence des modèles explicatifs psychologisants, la préoccupation pour les problèmes reliés à l'adaptation ou l'insertion sociale des individus, les politiques sociales de maintien à domicile et de désinstitutionnalisation semblent indiquer, d'une part, un retour en arrière dans l'explication des problèmes sociaux et, d'autre part, un désengagement progressif de l'État du champ social. Pour tenter d'éclairer ces questions, l'auteur reprend des données d'une enquête réalisée auprès de chercheurs québécois associés à la recherche appliquée et constate, au bout d'une analyse critique, que la recherche produit actuellement des connaissances " soit trop générales ou descriptives, soit des connaissances hyperspécialisées et fragmentées ", empêchant ainsi l'élaboration d'un savoir intégré sur les problèmes sociaux. Tout cela l'amène à conclure que même au lendemain du Rapport Rochon, le débat sur le rôle et la place de la recherche sociale en matière d'analyse et de solution aux problèmes sociaux est encore à faire. 
Richard Lefrançois, professeur au Département de service social, Université de Sherbrooke.

\section{Lecture de la recherche sur les problèmes sociaux}

Richard Lefrançois

Cet article propose une lecture critique visant à mieux comprendre les représentations de la recherche dans le champ des problèmes sociaux québécois. Cette réflexion s'appuie essentiellement sur une analyse, amorcée il y a une dizaine d'années et portant sur le rôle et les fonctions de la recherche sociale, ainsi que sur les données d'une mini-enquête que nous avons effectuée auprès d'un échantillon de chercheurs s'intéressant aux problèmes sociaux. Sans constituer un matériel empirique absolument sûr, ces données contiennent par ailleurs des indications intéressantes qui sont de nature à alimenter notre réflexion; aussi est-ce dans cette perspective que nous les utilisons dans le présent article.

Les discours contemporains sur les problèmes sociaux portent indubitablement la marque des changements structurels qu'a subis notre système de distribution de services sociaux. Sous l'impulsion de l'idéologie néo-conservatrice, des conceptions renouvelées de l'intervention et des problèmes sociaux se dessinent, marquées par un nouveau partage des responsabilités individuelles, familiales, communautaires et étatiques dans le processus de prise en charge des assistés.

La question examinée dans cet article se formule ainsi : en tant que champ traversé par des déterminations politiques, professionnelles et scientifiques, la recherche sur les problèmes sociaux est-elle appelée à se redéfinir à la lumière d'une dynamique transformée de la marginalité et de l'exclusion sociales, et dans le contexte d'un partenariat élargi ?

Eu égard à l'orientation de la recherche sociale, plusieurs éléments donnent à penser que l'on assiste, du point de vue du sens et de l'intentionnalité des actions sociales, à un retour en arrière :

- les schémas explicatifs psychologisants, ou le « recentrage sur la personne » (Robert, $1989: 41$ ), ont tendance à balayer les 
analyses mettant en cause les fondements des inégalités sociales;

- les problèmes sociaux sont interprétés presque exclusivement sous l'angle des phénotypes, à partir donc des difficultés d'adaptation et d'insertion sociale propres aux individus (Poirier, 1988 : 19);

- les défavorisés et les marginaux sont désignés comme s'ils formaient des catégories sociales inertes, plus ou moins étanches, et non des groupes sociaux actifs ou des fractions socialement déclassées;

- la société tend progressivement vers des consensus politiques qui masquent les contradictions et paralysent l'action des mouvements sociaux.

La recherche sociale semble en effet emboîter le pas, happée par les politiques régulatrices de l'État qui intensifient les services de maintien à domicile et canalisent les énergies vers de nouvelles pratiques d'intégration, de réadaptation et de soutien à l'endroit des personnes socialement exclues ou inadaptées. La désinstitutionnalisation implique notamment qu'une partie du fardeau de l'assistance soit transférée aux familles, aux groupes d'entraide et de support, bref à tout le réseau communautaire. Dans son discours officiel, l'État promulgue avec force l'idée qu'il faut prévenir ou freiner la dépendance institutionnelle, en créant de meilleures facilités d'adaptation à l'intention des personnes démunies physiquement, socialement ou économiquement, pour qu'elles puissent s'intégrer dans leur cadre de vie naturel.

Ces bouleversements ont justifié l'apparition d'un nouveau contrat social qui s'exprime par la volonté de l'État de se désengager de certaines responsabilités, celui-ci étant guidé essentiellement par des impératifs économiques. Par ailleurs, on pourrait penser que ce virage traduit au contraire le souci d'améliorer les conditions de vie des mésadaptés, en favorisant le renforcement des réseaux naturels qui paraissent mieux aptes à assurer leur pleine intégration et épanouissement ? Or, comment se situe la recherche sociale face à ces nouveaux partenaires de l'action sociale - groupes bénévoles, mouvements sociaux, communautés locales, familles - qui ont à relever le défi, en assurant le relais de manière efficace, conformément aux aspirations et velléités de multiples interlocuteurs ?

Paradoxalement, malgré ces efforts visant à minimiser les coûts de l'assistance, l'horizon des clientèles cibles s'élargit, sous l'effet de l'extension de la notion de besoin et de l'action combinée des capacités accrues de dépistage des populations à risque et du poids que représentent les nouveaux arrivants sur le marché de l'aide. Il y a là un effet pervers qui s'explique par le fait que les professionnels et 
les gestionnaires des programmes sociaux ont créé des espaces d'intervention et des champs de compétence qu'il est dans leur intérêt de protéger, assurant ainsi la légitimité de leurs actions et le maintien de leurs prérogatives.

Cette lecture rapide nous conduit donc à un autre questionnement : quelles transformations la nouvelle trajectoire qu'emprunte la société, à l'égard des plus démunis, fait-elle subir aux schèmes de représentations des problèmes sociaux que véhiculent les chercheurs ? Pour tenter de répondre à cette question, nous examinerons dans un premier temps les représentations que véhiculent un échantillon de chercheurs à propos des finalités de la recherche sociale appliquée, puis nous essaierons, dans un second volet, de proposer une interprétation générale.

\section{Les représentations de la recherche sociale appliquée}

Le développement récent de la recherche sociale appliquée (RSA), qualifiée ailleurs de l'Autre recherche (Joubert, Lacroix et Tremblay, 1984), semble s'inscrire dans une nouvelle perception de la pratique professionnelle, des problèmes sociaux et de la recherche elle-même. Afin de mieux comprendre quelles orientations se donne la recherche sociale appliquée, nous avons réalisé (Lacroix et Lefrançois, 1987) ${ }^{1}$ une enquête d'opinion auprès d'un échantillon de chercheurs $(\mathrm{N}=46$; échantillon par choix raisonné) œuvrant soit dans les universités $(N=22)$, soit dans le réseau de la santé et des affaires sociales $(N=24)$. Nous produisons dans les pages qui suivent les résultats les plus pertinents de cette recherche en lien avec la question qui nous préoccupe. Certains tableaux comparent les répondants selon qu'ils sont rattachés aux établissements de santé et de services sociaux (SS), ou aux institutions d'enseignement et de recherche $(\mathrm{RE})$. Les tableaux rapportent les scores moyens selon une échelle d'évaluation de type Likert :

$\begin{array}{ll}\text { SCORE } & \text { ÉVALUATION } \\ 1 & \text { Énormément } \\ 2 & \text { Beaucoup } \\ 3 & \text { Moyennement } \\ 4 & \text { Faiblement } \\ 5 & \text { Nullement }\end{array}$

À la question : Indiquez la contribution des objectifs suivants au développement de l'Autre recherche (la recherche sociale appliquée), la distribution des réponses s'établit comme suit (voir tableau 1) : 


\section{TABLEAU 1}

\section{Perception des objectifs de développement de la RSA \{scores moyens et (écarts-types) $\}^{*}$}

\begin{tabular}{|l|c|c|c|}
\hline \multicolumn{1}{|c|}{ OBJECTIFS } & TOTAL & SS & RE \\
\hline $\begin{array}{l}\text { Étude de l'impact social d'une mesure } \\
\text { ou d'un programme }\end{array}$ & $\begin{array}{c}1.63 \\
(.76)\end{array}$ & $\begin{array}{c}1.46 \\
(.66)\end{array}$ & $\begin{array}{c}1.79 \\
(.82)\end{array}$ \\
\hline Étude de satisfaction des besoins & 2.09 & 1.91 & 2.25 \\
$(.97)$ & $(.85)$ & $(1.05)$ \\
\hline $\begin{array}{l}\text { Étude de l'efficacité institutionnelle } \\
\text { d'une mesure }\end{array}$ & $\begin{array}{c}2.22 \\
(.99)\end{array}$ & $\begin{array}{c}2.14 \\
(.99)\end{array}$ & $\begin{array}{c}2.29 \\
(.98)\end{array}$ \\
\hline $\begin{array}{l}\text { Étude opérationnelle coûts et béné- } \\
\text { fices }\end{array}$ & $\begin{array}{c}2.76 \\
(1.14)\end{array}$ & $\begin{array}{c}2.71 \\
(1.29)\end{array}$ & $\begin{array}{c}2.79 \\
(1.0)\end{array}$ \\
\hline
\end{tabular}

* Les différences entre les deux sous-groupes ne sont pas significatives au seuil alpha .05 (test T) pour l'ensemble des items.

Les scores moyens enregistrés sur l'ensemble des items suggèrent que tous ces objectifs comptent pour beaucoup dans le développement de la RSA. Le lecteur aura noté que les descriptions proposées impliquent, dans la majorité des cas, le recours soit à la stratégie de la recherche évaluative ou opérationnelle, soit à celle de l'analyse exploratoire ou descriptive. Mais ce tableau révèle que les chercheurs, quel que soit leur milieu de rattachement, semblent davantage conscients que la RSA doit se développer en premier lieu en tenant compte des besoins de la clientèle, de préférence à ceux des établissements.

Un second tableau rapporte les scores obtenus en réponse à la question suivante : En vous référant à la méthodologie classique telle qu'enseignée à l'université ou dans les manuels, croyez-vous que l'Autre recherche accorde trop ou peu d'importance à chacun des aspects suivants? (voir tableau 2).

Ce tableau fait ressortir de façon convaincante ce qui semble perçu comme étant la spécificité de la RSA. Elle se situerait en quelque sorte aux antipodes de la recherche académique. La rentabilité immédiate, l'urgence de l'analyse, la pertinence institutionnelle prédominent sur les aspects de désignation reliés à la méthodologie, à la clarté du mandat, aux considérations théoriques et techniques de même qu'à la formation des chercheurs. 


\section{TABLEAU 2}

Appréciation des principaux éléments servant à qualifier la RSA $\{\text { scores moyens et (écarts-types) }\}^{*}$

\begin{tabular}{|l|c|c|c|}
\hline \multicolumn{1}{|c|}{ ITEMS } & TOTAL & SS & RE \\
\hline L'applicabilité à court terme & 2.37 & 2.67 & 2.09 \\
& $(1.20)$ & $(1.08)$ & $(1.24)$ \\
\hline Le temps de réalisation & 2.83 & 2.62 & 3.05 \\
& $(.99)$ & $(.90)$ & $(1.04)$ \\
\hline Le lien avec les décideurs & 2.84 & 2.86 & 2.82 \\
& $(1.22)$ & $(1.18)$ & $(1.23)$ \\
\hline Les priorités de l'État & 2.89 & 2.91 & 2.86 \\
& $(1.17)$ & $(1.08)$ & $(1.25)$ \\
\hline Les aspects techniques & 3.02 & 3.00 & 3.05 \\
& $(.70)$ & $(.76)$ & $(.64)$ \\
\hline La rigueur méthodologique & 3.02 & 3.14 & 2.91 \\
& $(.92)$ & $(.87)$ & $(.95)$ \\
\hline La clarté du mandat initial & 3.12 & 3.05 & 3.18 \\
& $(1.02)$ & $(.90)$ & $(1.11)$ \\
\hline La formation du chercheur & 3.18 & 2.96 & 3.41 \\
& $(.91)$ & $(.93)$ & $(.83)$ \\
\hline L'aspect théorique & 3.33 & 3.19 & 3.48 \\
& $(.94)$ & $(1.01)$ & $(.85)$ \\
\hline
\end{tabular}

* Les différences entre les deux sous-groupes ne sont pas significatives au seuil alpha .05 (test T) pour l'ensemble des items.

Examinons maintenant les représentations des fonctions de la RSA. Cette question fut posée aux répondants : Indiquez, selon vous, dans quelle mesure les fonctions suivantes caractérisent actuellement l'Autre recherche (voir tableau 3).

Ce tableau met en évidence que les chercheurs valorisent une pluralité de fonctions de recherche. Tous les scores moyens se situent largement dans le continuum des appréciations positives. Bien que ces items soient peu discriminants, on note tout de même, dans les deux sous-groupes, que les répondants ont tendance à reconnaître la 


\section{TABLEAU 3}

\section{Représentations des fonctions de la RSA \{moyenne (écarts-types)\}}

\begin{tabular}{|l|c|c|c|}
\hline \multicolumn{1}{|c|}{ FONCTIONS } & TOTAL & SS & RE \\
\hline L'identification des besoins & 1.89 & 1.87 & 1.91 \\
& $(.89)$ & $(.85)$ & $(.93)$ \\
\hline Le repérage de clientèles cibles & 2.02 & 2.13 & 1.91 \\
& $(.83)$ & $(.80)$ & $(.85)$ \\
\hline $\begin{array}{l}\text { Le support aux groupes ou associations } \\
\text { du milieu }\end{array}$ & 2.07 & 1.96 & 2.17 \\
\hline L'évaluation de programmes & $.93)$ & $(.88)$ & $(.96)$ \\
\hline Éclairer la prise de décision & 2.13 & 2.09 & 2.17 \\
& $(.96)$ & $(.95)$ & $(.96)$ \\
\hline L'expérimentation sociale & 2.13 & 2.23 & 2.04 \\
& $(.93)$ & $(.99)$ & $(.86)$ \\
\hline L'explication des problèmes sociaux & 2.18 & 1.86 & 2.48 \\
& $(1.12)$ & $(.92)$ & $(1.21)$ \\
\hline La critique sociale & $(1.28 *$ & 1.91 & 2.65 \\
& $(.88)$ & $(1.13)$ \\
\hline L'éducation populaire & 2.32 & 2.09 & 2.54 \\
& $(1.05)$ & $(1.02)$ & $(1.04)$ \\
\hline
\end{tabular}

* La différence entre les deux sous-groupes est significative au seuil alpha .02 pour cet item seulement.

nécessité d'arrimer la RSA aux opérations d'identification de besoins, de repérage des clientèles cibles et de support aux groupes du milieu. La critique sociale, l'éducation populaire et l'explication des problèmes sociaux ont un peu moins d'importance aux yeux des répondants.

Ces représentations des finalités de la RSA se transposent également dans les préférences des chercheurs en ce qui a trait aux stratégies de recherche sociale. Ainsi les études descriptives et exploratoires sont nettement priorisées au détriment des stratégies 
longitudinales de type expérimental ou des stratégies transversales utilisées dans l'étude des problèmes sociaux.

Comment donc interpréter globalement ces résultats qui suggèrent qu'une nouvelle vogue de la recherche sociale se dessine, celle qui privilégie le travail " clinique ", l'identification des besoins et les évaluations d'impact ? Quelles retombées théoriques et méthodologiques vont résulter de cette mutation des pratiques scientifiques qui se donnent plus que jamais comme objet le "traitement " des problèmes sociaux ?

II semble que les pressions exercées pour rentabiliser les productions scientifiques se feront de plus en plus vives, réduisant souvent la recherche à un rôle de support technique aux décisions et aux interventions programmées. Déjà, on assiste à la fois à un assujettissement et à un émiettement des efforts de recherche. La recherche sur les problèmes sociaux se ramifie ainsi en autant de champs que le système exige et qu'il peut supporter, qu'il s'agisse de développer les nouvelles problématiques de recherche touchant la toxicomanie, les minorités ethniques, les autochtones, les jeunes chômeurs, le vieillissement de la population, les familles monoparentales, la violence dont sont victimes les femmes, les enfants, les vieillards. Dans la même veine, la recherche est appelée à participer à l'édification d'un projet de société caractérisé par une harmonisation des rapports entre l'homme et son environnement et une meilleure qualité de vie. Pourtant, trop d'études concentrent davantage leur regard sur les effets de la dégradation des milieux de vie que sur les sources à la base de ces problèmes. Tel est à première vue le cadre dans lequel semble s'articuler le discours scientifique sur le social et où se mobilisent les énergies.

Or la recherche sociale doit-elle s'inscrire résolument dans une telle perspective qui, de notre point de vue, dilue de manière anarchique les responsabilités de la prise en charge des personnes en difficulté de fonctionnement social ? Au terme d'une analyse comparative sur les trajectoires québécoises et françaises de la recherche sociale (Lefrançois et Soulet, 1983), nous souhaitions élargir le débat à la lumière de ces trois observations majeures :

- la recherche sociale s'institutionnalise au rythme d'une plus grande efficacité et rentabilité de la mission de l'État dans la gestion des problèmes sociaux;

- I'accent est mis sur l'analyse des problèmes sociaux sous l'angle étroit des populations cibles identifiées par les différents partenaires du traitement individuel et de l'action sociale;

- les cheminements de recherche, tant théoriques que méthodologiques, sont éclatés et s'articulent autour d'un ensemble disparate de pratiques sociales. 
Le premier point souligne avec force le caractère utilitaire de la recherche sociale, la pertinence des thèmes d'étude se conjuguant avec les critères d'excellence dans l'espoir d'optimiser le rendement des travaux de recherche en termes de retombées scientifiques immédiatement transposables dans la pratique ou la décision politicoadministrative. Le second constat traduit la mise en service de l'appareillage de la recherche dans le champ des interventions sur le social, ainsi que le nouvel engagement des chercheurs dans la sphère de la définition et de la résolution des problèmes sociaux. Enfin, la dernière observation renvoie à l'état de sous-développement et de fragmentation des cadres de référence théoriques s'appliquant aux problèmes sociaux et à l'émergence de nouvelles approches méthodologiques (ex. : approches qualitatives).

Quelles peuvent donc être les difficultés pouvant résulter de I'implication de la recherche dans la gestion des problèmes sociaux?

\section{Les écueils appréhendés}

Depuis deux décennies, la recherche sociale n'est plus cloisonnée dans le champ constitutif du savoir scientifique. Elle est devenue une pratique sociale, vu son implication grandissante dans la poursuite des objectifs mis de l'avant par les établissements de santé et de services sociaux. Malgré la pénurie de chercheurs œuvrant dans les institutions du réseau, le message des organismes subventionnaires est clair et s'adresse à toute la communauté des chercheurs en sciences sociales: 1) Les efforts de la recherche doivent porter prioritairement sur l'étude des faits sociaux, non pas en tant que phénomènes à interpréter mais en tant que problèmes à résoudre. 2) Les chercheurs sont sollicités pour s'intégrer dans les milieux de pratique et ancrer leur réflexion sur les axes d'intervention définis par les programmes sociaux existants. Le volet «Étude et analyse » du programme de subvention à la recherche du CQRS (Conseil québécois de la recherche sociale) illustre bien cette volonté d'arrimer la recherche aux pratiques sociales développées ou soutenues par l'État. 3) Enfin, la transférabilité des connaissances scientifiques dans la pratique fait de plus en plus l'objet d'une préoccupation majeure.

On vise donc le développement d'une technologie sociale qui puisse répondre de façon pragmatique aux demandes administratives et aux politiques d'intervention des établissements du réseau. Dans cette perspective, deux modèles idéaux de chercheurs semblent émerger : 1) les agents de recherche généralistes à qui on soumet le mandat de dresser des profils descriptifs de clientèles, dépister des nouveaux besoins, colliger des informations, bâtir des outils et 
interpréter des données informatisées: tels sont les rôles du chercheur-technicien; 2) à l'autre extrême, les chercheurs plus expérimentés (ou mieux positionnés dans la structure hiérarchique) sont responsables de dossiers touchant l'évaluation des programmes, les études-bilans, les expérimentations sociales ou la conception de projets-démonstrations, divers mandats confiés aux chercheurs professionnels. Ces deux rôles convergent, semble-t-il, vers un même objectif : faire en sorte que la recherche sociale produise de la légitimation institutionnelle, qu'elle cautionne les pratiques et idéalement les alimente, et enfin qu'elle devienne une forme technique de contrôle social.

Une autre question dès lors surgit : le chercheur scientifique dispose-t-il encore de l'autonomie suffisante et du regard critique susceptible à la fois de contribuer socialement à la résolution des problèmes sociaux, et en même temps de produire un discours critique sur les problèmes sociaux? On s'étonne que la recherche sociale se soit peu penchée sur les mécanismes qui interviennent dans la fabrication d'un problème social, notamment ceux qui engendrent la stigmatisation des exclus ou des personnes dépendantes. Par exemple, il faut déplorer que peu de chercheurs québécois aient examiné les conséquences psychosociales pour la personne assistée du rapport bureaucratique et informel qui se développe dans la relation d'aide professionnelle. Les ethnométhodologues - surtout ceux ayant exploré le champ de la déviance (Lemert, 1967; Matza, 1964) - ont pourtant ouvert la voie à des perspectives d'analyse très fécondes pour le travail social. Ils ont montré par exemple que les dispositifs socio-institutionnels opérant dans le processus de la désignation sociale favorisent les dérives vers la marginalité et accentuent la confirmation du rôle de dépendant chez le bénéficiaire. Interrogeant à son tour les pratiques du travail social, Kurc (1985: 96) va même jusqu'à conclure : "Le travail social peut être aussi bien considéré comme la réponse aux problèmes sociaux que l'on s'attache à résoudre que comme un mécanisme de construction d'un problème social permettant aux travailleurs sociaux de trouver un accord sur l'édification de leurs normes."

Pour véritablement se développer, il semble donc que la recherche sociale doive se détacher, en partie du moins, du mode de pensée contraignant et limitatif qui anime les appareils chargés de la gestion des services sociaux. Qui plus est, une compréhension adéquate des problèmes sociaux appelle sans doute une logique d'analyse toute différente qui soit en mesure de saisir la dynamique institutionnelle même du problème social, c'est-à-dire en tant que construit. À défaut d'un tel élargissement du cadre de référence de la 
recherche sociale appliquée, certains écueils sont à appréhender, et il semble indiqué d'identifier les principaux :

- Le fait de réfléchir sur les problèmes sociaux uniquement dans l'optique des programmes sociaux et des modes d'intervention est sujet à occulter les déterminations politico-idéologiques et économiques à la base de ces problèmes. Les lectures partielles, locales, conjoncturelles ou très pointues sur les problèmes sociaux risquent ainsi de se substituer aux analyses plus pénétrantes les resituant dans leur propre historicité.

- Considérant le pouvoir politique énorme dont sont investis les appareils de gestion des problèmes sociaux, il est à craindre que la recherche sociale soit domestiquée au point qu'elle en vienne à participer aveuglément au projet de rationalisation et de structuration de l'action sociale. Concrètement, cela signifie que les problèmes sociaux ne seront reconnus ou officialisés qu'à la condition d'avoir reçu une légitimation institutionnelle et technocratique.

- En raison du trop grand morcellement des activités scientifiques et des préoccupations tous azimuts qui caractérisent déjà l'organisation de la recherche sociale, il est à se demander comment mettre en commun et synthétiser les résultats obtenus, comment intégrer les savoirs dans une optique globale et pluridisciplinaire.

- En devenant plus utilitaire, on peut redouter que la recherche sociale sanctionne un désintéressement pour le travail théorique ou critique et qu'elle s'interdise d'entrevoir d'autres logiques d'analyse, d'autres stratégies pour aborder l'étude des problèmes sociaux.

- Finalement, là où les demandes en expertise de recherche sont souvent très pressantes, où par conséquent le chercheur ne dispose pas du temps nécessaire à l'approfondissement ou au mûrissement de sa réflexion, la qualité méthodologique des productions scientifiques peut se dégrader, contribuant ainsi à disqualifier la recherche sociale appliquée.

Il faut donc regretter qu'à l'heure actuelle la recherche sociale produise des connaissances soit trop générales ou descriptives, soit des connaissances hyperspécialisées et fragmentées, ce qui rend difficile le développement d'un savoir intégré sur les problèmes sociaux. En revanche, la recherche sociale appliquée interpelle les sciences humaines à maints égards. Elle nous rappelle la nécessité de se pencher de manière concrète sur les problèmes actuels de civilisation. Elle signale que la recherche peut être mise à la disposition des défavorisés en vue d'améliorer leurs conditions existentielles. En outre, la recherche sociale appliquée a contribué au 
développement des méthodologies douces, à des stratégies de recherche-action pour mieux épauler les projets émanant de la base sociale.

La réflexion sur les problèmes sociaux est aujourd'hui nourrie par une pluralité d'acteurs engagés, à des degrés d'implication divers, dans la régulation des rapports sociaux. Législateurs, gestionnaires d'établissements et de programmes, intervenants psychosociaux, bénévoles, organisateurs communautaires ou chercheurs, tous participent d'une certaine manière à l'élaboration d'un discours sur le social. Or, devant l'ampleur des mutations découlant des récentes réformes des politiques et de l'aide sociales ainsi que des stratégies entourant la dispensation des services sociaux, peut-on conclure à l'existence d'une conception unifiée des problèmes sociaux à l'heure actuelle ? Un consensus se dégage-t-il sur les solutions à mettre en place pour juguler les inégalités socio-économiques et contribuer au mieux-être des différents groupes de citoyens? Plus fondamentalement, d'autres se demandent encore si notre compréhension actuelle des problèmes sociaux nous permet d'anticiper que des solutions à plus long terme, efficaces et durables, seront apportées dans l'optique du plein épanouissement des individus. Or, quelle place la recherche sociale occupe-t-elle, au lendemain du Rapport Rochon, dans l'élaboration d'un savoir capable d'alimenter la réflexion en matière de définition, $d^{\prime}$ 'interprétation et de solution aux problèmes sociaux ? Voilà autant de nouvelles questions à débattre.

\section{Note}

${ }^{1}$ Cette recherche a été réalisée dans le cadre d'un programme d'échange avec le CRTS (Centre de recherche sur le travail social) de l'Université de Caen. Le but de l'étude consistait à mettre en parallèle les représentations de la recherche chez les chercheurs québécois et français, en comparant la recherche académique et la recherche sociale appliquée.

\section{Références}

KURC, A. (1985). "Travail social : effets émergents, effets pervers ", POUR, $\mathrm{n}^{\text {os }}$ 103-104 (nov.-déc.) : 87-96.

Joubert, P., L. LACROIX et M.A. Tremblay (1984). L'autre recherche. Actes du colloque du $52^{\mathrm{e}}$ congrès de l'ACFAS, section "Santé communautaire ", Québec.

LACROIX, L. et R. LeFRANÇOIS (1987). L'autre recherche. Textes et matériel d'enquête (non publié). 
LEFRANÇOIS, R. et M.H. SOULET (1983). Le système de la recherche sociale, Tome 1 : La recherche sociale dans l'État, Collection Recherche sociale, $n^{\circ s} 3-4$. Sherbrooke.

Lemert, E.N. (1967). Human Deviance, Social Problem and Social Control. Englewood Cliffs : Prentice-Hall.

MAtZA, D. (1964). Delinquency and Drift. New York : Wiley.

POIRIER, C. (1988). La recherche sociale appliquée. Dossier thématique préparé pour la Commission d'enquête sur les services de santé et les services sociaux, Québec.

ROBERT, L. (1989). " Le partenariat entre le réseau institutionnel et la communauté : un paradigme à définir ", Nouvelles pratiques sociales, vol. 2, $\mathrm{n}^{\circ} 1: 37-52$. 